\title{
THE
}

$5-25-2010$

\section{Fusion Product Planning: A Market Offering Perspective}

\author{
Yuwen Chen \\ University of Rhode Island, yuwen@uri.edu \\ Janice E. Carrillo \\ Asoo J. Vakharia \\ Peter Sin
}

Follow this and additional works at: https://digitalcommons.uri.edu/cba_facpubs

This is a pre-publication author manuscript of the final, published article.

Terms of Use

All rights reserved under copyright.

\section{Citation/Publisher Attribution}

Chen, Y., Carrillo, J. E., Vakharia, A. J. and Sin, P. (2010), Fusion Product Planning: A Market Offering Perspective. Decision Sciences, 41: 325-353. doi: 10.1111/j.1540-5915.2010.00271.x. Available online at http://onlinelibrary.wiley.com/doi/10.1111/j.1540-5915.2010.00271.x.

This Article is brought to you for free and open access by the College of Business at DigitalCommons@URI. It has been accepted for inclusion in College of Business Faculty Publications by an authorized administrator of DigitalCommons@URI.For more information, please contact digitalcommons-group@uri.edu. 


\title{
Fusion Product Planning: A Market Offering Perspective
}

\begin{abstract}
Devices that integrate multiple functions together are popular in consumer electronic markets. Examples include the cellular phone that takes digital pictures and plays MP3's, the PDA with cell phone, and multi-function office machines. We describe these multi-function devices as fusion products since they fuse together products which traditionally stand alone in the marketplace. In this paper, we investigate the manufacturer's fusion product planning decision adopting a market offering perspective which allows us to address the design and product portfolio decisions simultaneously. The general approach adopted is to develop and analyze a profit maximizing model for a single firm which integrates product substitution effects in identifying an optimal market offering. In the general model, we demonstrate that the product design and portfolio decisions are analytically difficult to characterize since number of possible portfolios can be extremely large. To resolve this, we propose an algorithm which identifies the optimal solution and the corresponding product design.

The managerial insight from a stylized all-in-one model and numerical analysis is that the manufacturer should in most cases select only a subset of fusion and singlefunction products to satisfy the market's multi-dimension needs. This may explain why the function compositions available in certain product markets are limited. In particular, one of the key factors driving the product portfolio decision is the margin associated with the fusion products. If a single all-in-one fusion product has relatively high margins, then this product likely dominates the product portfolio. Also, the congruency of the constituent single function products is an important factor. A portfolio of single function products is considered to be fairly congruent if it is easy to create a fusion product from them and that the newly fused product is serving a similar market as the original single-function products. When substitution effects are relatively high (i.e. the product set is more congruent), a portfolio containing a smaller number of
\end{abstract}


products is more likely to be optimal. Conversely, when substitution effects are relatively low (i.e. the product set is more incongruent), then the optimal product portfolio is generally larger in size and more sensitive to small changes in profit margins.

Key words: fusion product, multifunction product, demand substitution, product portfolio, new product development

\section{INTRODUCTION}

Technology advances have made it possible for firms to offer a wider range of integrated products. For example, (i) an integrated printer, scanner, copier, and fax is commonly found on the shelves of most office product stores; (ii) a cell phone with PDA, gaming, and camera capabilities is offered by most cellular service providers; (iii) a digital camera and video recorder is available through most technology retailers; and (iv) an MP3 player which can serve as a thumb drive, a digital voice recorder, and a radio tuner is marketed as an all-in-one device. This feature integration is not an entirely new phenomenon since the Swiss Army knife has been around for over five decades and a radio tuner with an integrated cassette tape recorder was a commonly available product a few decades back.

Since the late 1990's, however, there has been a substantive increase in the number of products which integrate multiple functionalities. This is probably due to a variety of reasons. First, recent advances in integrated circuit design, interface standards, and wireless protocols have enabled the technological development of devices which integrate multiple functionalities (Rysavy, 2004). Second, the growing number of tech savvy users who prefer carrying a single product which seamlessly consolidates multiple functionalities (e.g., computing, organizing, communication, data storage, and gaming) has also led to an established market demand for these devices. Finally, from an individual customer perspective, these integrated devices might be preferred since they could be priced lower than the total cost of the constituent devices. 
In this paper, we refer to these integrated devices as fusion products (FPs). An alternative term might be multifunction products but this has been used in the past to refer to integrated office machines and hence, might be too restrictive. Further, according to the MerriamWebster dictionary, 'fusion' means "a merging of diverse, distinct, or separate elements into a unified whole" and hence, we feel the term fusion products is more representative of the newer integrated devices. In general, we define a fusion product as one which combines two or more functionalities in a single device. Most FPs are preceded in the marketplace by multiple single-function products (SPs).

Rust, Thompson, and Hamilton (2006) pointed out that firms are interested in integrating as many functionalities as possible in a single fusion product due to several reasons. First, the marginal cost of adding a new feature or an extra function costs little or even nothing, and thus, "engineers can’t resist the temptation to equip existing electronic components with more functions." Second, the firm is "aiming to hit two birds with one stone" in the sense that it would like to capture all consumers who are interested in each functionality through a single fusion product. Third, marketers believe that "more is better" and hence, adding another feature always makes the product more appealing. From the consumer perspective, Thompson, Hamilton, and Rust (2005) found that most consumers do perceive that "more is better" before they buy and use the FP. However, after consumers buy the FP, they frequently experience "feature fatigue."

Based on this discussion, it appears that a critical firm level decision is to identify the number of distinct functionalities which should be integrated into a single FP. On the one hand, the argument for including a larger number of functionalities within a single FP could be that technologically it might be possible to carry this out with relative ease and low marginal cost. Conversely, combining a fewer number of functionalities into a single FP might be preferred so as to avoid the "feature fatigue" phenomenon experienced by consumers.

The key managerial decisions which are addressed in this research are those that relate 
to product development and product introduction. Ulrich and Eppinger (2000) delineate the product development process into six distinct phases including: (i) Product Planning, (ii) Concept Development, (iii) System-Level Design, (iv) Detail Design, (v) Testing and Refinement, and (vi) Production Ramp-up. During the product planning phase, firms must answer questions concerning what mix of fundamentally new products, platforms and derivative products to bring to market. Key decisions made in the concept development and system level design phases include investigating feasibility of product concepts, building and testing of industrial prototypes, generating alternative product architectures, defining major subsystems and interfaces, and refining the industrial design. The outcome of the detailed design phase includes complete specifications for the geometry, materials and tolerances of all parts in the product, while the effectiveness of the product design is evaluated during the testing and refinement phase. Finally, during the production ramp-up phase, the product is transitioned to manufacturing and launched to the market. The model introduced in this paper links potential new technologies and functions to the specific portfolio of products incorporating these new functions. Consequently, this model can be utilized by decision makers to identify an appropriate fusion product portfolio during the product planning phase of the product development process. In this context, our focus is primarily on the composition of the product portfolio in the presence of product substitution effects (i.e., an FP might be viewed as a substitute for an SP). We focus specifically on examining the feasibility of product concepts (in terms of SPs and FPs) with a view to generating product plans which should be considered for the subsequent phases of design, testing, refinement, and production ramp-up.

Similarly, in the technology management literature, one of the key decisions associated with technology strategy is to identify which potential technologies should be included in the firm's portfolio of products (Burgelman, Christensen, \& Wheelwright, 1996). To illustrate, Fusfeld (1978) advocates (i) formulating a product/technology matrix which links each potential technology to each product in the firm's portfolio and (ii) identifying the firm's 
strength in each area. In this model, we are essentially mapping the firm's technologies with the portfolio of products based on these technologies. Specifically, a strategic level manager would utilize our model to gain insights concerning the FP portfolio problem after the firm's technologies have been identified and during the initial product planning phased of the development process. In particular, our model offers guidance concerning which of the technologies to combine into FPs, and how many products to bring to market based on these technologies.

The focus of this paper is to provide insights into three key managerial decisions: (i) FP design, (ii) the composition of the product portfolio, and (iii) anticipated quantities and prices for these products. From an FP design perspective, it is assumed that the firm has already identified alternative product functionalities (and related technologies) and is now confronted with the design problem of which of these technologies should be integrated into product offerings. From a product portfolio perspective, we are also interested in the set of distinct products the firm should combine into a portfolio of offerings. A unique feature of our paper is that we integrate product substitution effects as well as aggregate market demand in making both these managerial decisions. From a production and market planning perspective, we determine the optimal quantities and prices that the firm should target for each product in the portfolio.

The remaining part of this paper is organized as follows. In the next section, we review the relevant literature and research on FPs. In Section 3, we develop an economic model for addressing the decision as to which functionalities should be integrated when offering FPs to the market. In order to provide managerial insight from our model, we analyze a special case of our approach in Section 4; and we perform a numerical analysis to identify the impact of key parameters in making the design decision in Section 5. Finally, conclusions and managerial implications of this research are discussed in the Section 6. 


\section{RELEVANT LITERATURE}

Though many business and technology journals have paid attention to the phenomenon of product-fusion (Anonymous, 1997; Avery, 2004; Harbaugh, 1998; Magid, 1998; Meyers, 2004; Schonfeld, 2004; Walker, 2004), there are still very few academic, theoretical papers discussing this subject. Thompson et al. (2005) investigated consumer's feature fatigue while purchasing these types of products. Their results are that consumers have a higher evaluation of a product with more features prior to the purchasing decision. However, after use, consumers find that there is a negative association between product usability and the number of features integrated in the product.

Chen, Vakharia, and Alptekinoğlu (2008) investigate the product portfolio decision for a firm which can offer two single-function products and one multi-function (two-function) product to the market. Given this setting, they focus on identifying key parameters which drive the decision to either include or not include the multi-function product in a product portfolio. Our paper extends this analysis to a more general context where the number of functionalities available for a fusion product is significantly larger. This allows us to examine issues related to FP design where we determine which functionalities should be integrated into a single FP. In addition, we also allow for asymmetric substitution effects between products since we feel that this is more representative of industry practice. Given this general setting, we also show that the FP design and product portfolio decisions are significantly more complex which leads to the design of an efficient algorithm for addressing both these decisions.

Other relevant literature can be classified in one of two categories: research on product variety and product line selection; and research on product bundling and vertical differentiation. 


\subsection{Product Variety and Product Line Selection}

According to Lancaster (1990), product variety refers to the number of variants within a specific product group. There are four different views concerning product variety: the individual consumer, the individual firm, market equilibrium, and the social optimum. Product variety may depend on the competitive ecology of the industry and become less valuable when the total number of products in the market increases (Sorenson, 1999). Ramdas and Sawhney (2001) propose an optimization model which can identify a subset of line extensions that has maximum incremental profits. Loch and Kavadias (2002) recognize the inherent combinatorial complexity of optimally determining a project portfolio when analyzing this decision from the first stage technology development and R\&D investment level. They focus on the dynamic allocation of resources over a fixed planning horizon which can guide managers in the development of a new product with several associated product lines.

Papers which have addressed the product line selection and pricing problem are those of Dobson and Kalish (1988); Chen and Hausman (2000); and Hopp and Xu (2005). A second set of product assortment models utilizes the multinomial logit (MNL) choice model to estimate consumer utilities and purchasing choice. Cachon, Terwiesch, and Xu (2005) investigate the retailer's assortment problem when consumer search is possible. They differentiate three models of consumer search: no search, independent assortment search and operlapping independent assortment. In some cases, the optimal assortment is within a defined popular set of products for no search and independent search models. Other papers also using MNL choice models are Aydin and Ryan (2000); Smith and Agrawal (2000); van Ryzin and Mahajan (1999); and Mahajan and van Ryzin (2001).

\subsection{Product Bundling and Vertical Differentiation}

Product bundling was first suggested by Stigler (1968), who viewed it as a strategy for a monopolist to utilize price discrimination under the assumption that heterogeneous consumers have different willingness to pay (reservation values). Early research in the area investigated 
issues related to the optimal strategy of sellers, consumer surplus and the effects on competition (Adams \& Yellen, 1976; Dansby \& Conrad, 1984; Schmalensee, 1982; Schmalensee, 1984; McAfee, McMillan, \& Whinston, 1989; Hanson \& Martin, 1990). Some marketing oriented research focuses on retail or information goods bundles is presented in Mulhern and Leone (1991) and Bakos and Brynjolfsson (2000). Even though knowledge of the consumer's utility function is a widely accepted assumption in bundling analysis, the method how consumers judge, perceive, and evaluate the bundle deal were not studied until recently (Yadav \& Monroe, 1993; Kaicker, Bearden, \& Manning, 1995; Simonin \& Ruth, 1995).

In vertical differentiation models, the focus is on the market for a base product with multiple variants that differ along one single quality dimension (for product variants that differ on two quality dimensions, see Baumol, 1967; Vandenbosch \& Weinberg, 1995). These approaches assume consumers have the same ranking of the variants of this product and thus, if all variants are equally priced, consumers will choose the best quality product. The heterogeneity of consumers can be on income budget distribution (Baumol, 1967; Gabszewicz \& Thisse, 1979) or on quality preference distribution. Most models assuming difference in

quality preference also assume that the distribution is uniform (Moorthy, 1984; Vandenbosch \& Weinberg, 1995; Wauthy, 1996). Ansari, Economides, and Ghosh (1994) relaxed the quality preference distribution to a generalized beta distribution, yielding substantially different results from those assuming uniform distribution. Two papers investigated vertical differentiation over time under models of intertemporal competition (Moorthy \& Png, 1992; Deneckere \& de Palma, 1998). Rather than review this extensive body of literature in further detail, we refer the reader to Kaul and Rao (1995).

\subsection{Summary}

The notion of product variety applied in this paper is different from that of Lancaster (1990). Product variety and product portfolio issues in this paper indicate the number of variants 'across' several product groups for an individual firm which can offer both single function 
and fusion products. Different from MNL choice model approaches, this paper focuses on the aggregate demand and identifies FP designs which are targeted toward the entire market rather than an individual consumer.

A fusion product is quite different from a bundled product on a number of dimensions. First, the cost of producing a fusion product can be greater or less than the sum of all costs of its component products. In general, this cost is a function of the level of integration associated with combining multiple functionalities. Second, from an individual consumer perspective, the reservation values for fusion products do not necessarily equal the sum of reservation values of its component products. Third, functional integration tends to make fusion products substitutes for existing products while bundled products are usually complementary. Although, a fusion product can be seen as a high-end version of its component products, this view ignores the impacts on the sales of other single-function products.

In sum, this paper aims to fill a substantive void in current research on FP design. In addition, our approach in addressing this design decision is unique in the sense that we adopt a market offering perspective and thus, are simultaneously able to incorporate demand effects in product design. In the next section, we present an analytical model to address the key design issue of how many functionalities should be included in each FP introduced to the market.

\section{MODEL DEVELOPMENT AND ANALYSIS}

\subsection{Preliminaries}

Assume there exists a product-fusion technology that can integrate any combination of functions 1 to $n$ into FPs. As a result, the firm has the capability to offer $m=2^{n}-1$ different products which includes $n$ products each with a single functionality and $(m-n)$ FPs. Instead of directly addressing the problem of how many functionalities should be combined when designing FPs, we approach this issue indirectly using a product portfolio perspective. 
In essence, we focus on identifying an optimal product portfolio (which includes at most $m$ products) and note that by examining the components of such a portfolio, the firm can identify which functionalities should be incorporated in each product. Our contention is that such an approach is more comprehensive since it provides input into the design decision (for FPs) by integrating product substitution effects and market demands.

When the fusion-technology makes FPs possible, then the demands for all products are more dynamic due to substitution effects. If any two of these $m$ products possess similar functionality, then there exists some degree of substitution between the two markets for these products. Using the manufacturer of office machines as an example, single-function products which could potentially be offered are the fax machine, copier, printer, and scanner (i.e., $n=4$ ). Given no overlap in functionalities between each of these products, they are not considered substitutes. With the availability of fusion-technology, the manufacturer now has the capability of offering 11 FPs (i.e., $\left.2^{4}-(4+1)\right)$ and depending upon the functionalities included in each of them, these could be considered substitute products. For example, assume that the manufacturer introduces an FP which integrates the functionalities associated with a printer and a copier. In this case, this product would be a substitute for the single function printer, the single function copier, and other FPs which incorporate the functionality of a printer and/or a copier (e.g., printer/fax; copier/fax; printer/copier/fax).

To differentiate various levels of combinations of function, in this paper, a fusion device equipped with all functions, is called all-in-one, and a fusion device with only some functions is called some-in-one. For example, consider a four-function set containing functions of copying, scanning, printing and fax. Then, a copier/printer and a printer/copier/fax are examples of some-in-one, and a device with four functions is an example of an all-in-one product. Due to the complexity of the model, we assume that the fusion technology is exogenously given.

Before specifying the inverse demand function, we first describe substitution effects. The substitution among $m$ products can be represented by a $(m \times m)$ asymmetric substitution 
matrix $r$ :

$$
r=\left[\begin{array}{ccccc}
1 & r_{1,2} & r_{1,3} & \cdots & r_{1, m} \\
r_{2,1} & 1 & r_{2,3} & \cdots & r_{2, m} \\
r_{3,1} & r_{3,2} & 1 & \cdots & r_{3, m} \\
\vdots & \vdots & \vdots & \ddots & \vdots \\
r_{m, 1} & r_{m, 2} & r_{m, 3} & \cdots & 1
\end{array}\right]
$$

where $0 \leq r_{k, j}<1(k \neq j)$. A small (large) $r_{k, j}$ is associated with weak (strong) substitution effect of product $k$ substituted by product $j$. These product substitution effects reflect the overlap between two different product markets, but are influenced by the independent functions contained within the specific product. If product $k$ and product $j$ have a common function, then it is likely that there will be some overlap in the product markets such that $0<r_{k, j}<1$. If, on the other hand, there is no function commonality between two products $k$ and $j, r_{k, j}$ is assumed to be zero.

Note that, different from Chen et al. (2008), the substitution between any two products in this model can be asymmetric (Sethuraman, Srinivasan, \& Kim, 1999; Allenby \& Rossi, 1991). Normally, a high-end product has a stronger substitution effect to its low-end substitute than vice-versa. For example, an all-in-one printer has stronger substitution effect on the single function printer than the converse. Managers can utilize the estimation techniques shown in Ben-Akiva and Gershenfeld (1998) and Hendel (1999) for assessing the substitution matrix for their firm. Also, a technique similar to that described in Chen and Hausman (2000) for choice-base conjoint analysis can be adapted to derive aggregate demand level parameters.

Market demands for each of the $m$ products with substitution effects are represented through linear inverse demand functions which have commonly been used in the literature (Singh \& Vives, 1984; Li \& Zhang, 1999; van Mieghem \& Dada, 1999; Dobson \& Yano, 2002; Dasci \& Laporte, 2004; Pekgun, Griffin, \& Keskinocak, 2005). Because no two products are exactly identical to each other, we assume each product $k(k=1, \ldots, m)$ has its own 
maximum price $a_{k}$. Then for each product $k$, if $p_{k}$ represents the market price, and $q_{k}$ the quantity offering to the market, we define $a, p$, and $q$ be the $(m \times 1)$ vectors of maximum price, market price, and quantity offerings, respectively. Based on this, the inverse demand functions are as follows:

$$
p=a-r q
$$

Assuming that the variable cost per unit associated with product $k$ is $c_{k}$ and $c$ is the $(m \times 1)$ unit variable cost vector with the $k^{t h}$ element defined as $c_{k}$. Then the profit function of the firm is

$$
\begin{aligned}
(G P): & \Pi=q^{T}(p-c)=q^{T}(a-c-r q)=q^{T}(2 d-r q) \\
\text { s.t. } \quad & q \geq 0 .
\end{aligned}
$$

where $d$ is the maximum profit margin $(m \times 1)$ vector with the $k^{t h}$ element defined as $d_{k}=0.5\left(a_{k}-c_{k}\right)$. We do not include the fixed cost in the objective function since we assume the investment in technology has already been made and thus, there is no additional fixed cost of function combination selection. Next, we analyze this model to obtain some key insights. The variable notation used in this paper is summarized in Table 1.

$$
\text { Insert Table } 1 \text { here }
$$

\subsection{Analysis}

Due to asymmetric substitution effects, designing and offering all combinations of FPs might not be an optimal strategy for the firm. From a practical perspective, this is one explanation for why a technologically feasible product such as a printer with only a faxing functionality is not offered in the market place. Of course, the key managerial issue is whether such industry practices can be replicated in our model with a view to providing some face validity. Given that the firm's objective is to identify the optimal product portfolio and the quantity offerings for each product in the portfolio which maximizes the firm profits, we analyze this problem 
further. Given $m\left(=2^{n}-1\right)$ technologically feasible products, the firm needs to choose between $2^{2^{n}-1}-1$ product portfolios.

Let us first start by evaluating whether the objective function to model GP is strictly concave in the decision variables. In order to do this, we note that the Hessian (see Appendix A) is defined as:

$$
H=(-2)\left[\begin{array}{ccccc}
1 & \gamma_{1,2} & \gamma_{1,3} & \cdots & \gamma_{1, m} \\
\gamma_{1,2} & 1 & \gamma_{2,3} & \cdots & \gamma_{2, m} \\
\gamma_{1,3} & \gamma_{2,3} & 1 & \cdots & \gamma_{3, m} \\
\vdots & \vdots & \vdots & \ddots & \vdots \\
\gamma_{1, m} & \gamma_{2, m} & \gamma_{3, m} & \cdots & 1
\end{array}\right]=(-2) \gamma
$$

where $\gamma_{k, j}=\frac{1}{2}\left(r_{k, j}+r_{j, k}\right)(k, j \in\{1,2, \ldots, m\}$ and $k \neq j)$ represents the average substitution effect between products $k$ and $j$. To establish concavity, we need to show that the principal minors of $H$ alternate in sign. Although this can be easily shown for the case of $n=2$ functionalities with some additional restrictions on the substitution effects (Chen et al., 2008), it is analytically difficult to reach this conclusion when FPs can be designed with $n \geq 3$ functionalities. Also note that, in some cases, concavity of the objective function does not guarantee that by simultaneously solving the FOC, we can determine the optimal quantities since such an interior solution might violate the non-negativity constraints on the these decision variables.

Based on this, we start by formulating the Lagrangian for our profit maximizing model as follows:

$$
(G L): \quad \Pi_{G L}=q^{T}(p-c)=q^{T}(2 d-r q)+q^{T} \nu
$$

where $\nu_{k}$ is the lagrangian multiplier of quantity $q_{k}(k=1, \ldots, m)$ and $\nu$ is the lagrangian multiplier vector. The FOCs for this model which identify necessary conditions for optimality) lead to the following solution for the quantity offering and lagrangian multiplier vectors 
(see Appendix B):

$$
\begin{aligned}
& q=[\gamma]^{-1}\left(d+\frac{1}{2} \nu\right) \\
& \nu \geq 0
\end{aligned}
$$

and, of course, for each product offering $k, q_{k} \nu_{k}=0$. This leads to some interesting insights. For some product offering $k$, if $q_{k}>0$, then we have $\nu_{k}=0$; otherwise, when $\nu_{k}=2\left(\sum_{j=1, j \neq k}^{m} \gamma_{k, j} q_{j}-d_{k}\right)>0, q_{k}=0$. It is also possible that both the quantity offering and the lagrangian multiplier are zero simultaneously, which occurs when there is a boundary solution.

The content of $\nu_{k}$ implies that a product $k$ with a "relatively small" profit margin is more likely to have a positive lagrangian multiplier and hence, not be part of the product portfolio while the product with a "relatively large" profit margin is more likely to be included in the product portfolio. However, a high profit-margin product may not be selected if the substitution effects with other products are too strong. Thus, the market finding that certain technologically feasible fusion products might not be offered by a firm is also validated through an analysis of the lagrangean multiplier $\nu_{k}$. In addition, this type of analysis also resonates with some of the results in Chen et al. (2008) where the substitution effects were assumed to be symmetric.

Now let us identify the optimal product offerings. In order to do this, we first define a product portfolio $S$ as one which consists of specific non-zero quantity offerings for each product included in the portfolio and also has an associated profit function which is concave. For such a given portfolio, let $d_{S}$ be the maximum scaled profit margin vector, $q_{S}$ be the quantity offering vector, $r_{S}$ be the substitution effects vector, and $\gamma_{S}$ the average substitution effects vector. Then, it is easy to show that the non-zero quantity offering vector $q_{S}$ and the 
associated profit for a given portfolio $S$ are (see Appendix C):

$$
\begin{aligned}
q_{S} & =\left[\gamma_{S}\right]^{-1} d_{S} \\
\Pi_{S} & =q_{S}^{T} r_{S}^{T} q_{S} \\
& =\frac{d_{S}^{T} \operatorname{Adj}\left[\gamma_{S}\right] d_{S}}{\left|\gamma_{S}\right|}
\end{aligned}
$$

In case of symmetric substitution effects, the non-zero quantity offering vector $q_{S}$ and the associated profit can be restated as:

$$
\begin{aligned}
q_{S} & =\left[r_{S}\right]^{-1} d_{S} \\
\Pi_{S} & =\frac{d_{S}^{T} \operatorname{Adj}\left[r_{S}\right] d_{S}}{\left|r_{S}\right|}
\end{aligned}
$$

when we have symmetric substitution effects.

\subsection{Dominant Portfolios}

With $m$ potential products, there are theoretically $2^{m}-1$ distinct portfolios (e.g., with 3 functionalities, $m=2^{3}-1=7$ products and thus, $2^{7}-1=127$ potential product portfolios) of this type. Given that the number of distinct portfolios is substantially large, we develop analytical results which can help to pare down the number of potential portfolios which could be potentially optimal. Let $S$ be a product portfolio of $s$ products. If $S^{\prime}=S \cup\{j\}$ and

$j \notin S$, then we call $S^{\prime}(S)$ the parent (child) portfolio of $S\left(S^{\prime}\right)$. Constructing the hierarchy of product portfolios, we know that a portfolio consisting of all m products is at the highest level of parent portfolios since it contains all possible product variants in a single portfolio. In contrast, one-product portfolios are the lowest level of child portfolios. A parent portfolio with $i$ products contains $i$ direct children portfolios, such that each child portfolio has one product less than its direct parent. For example, if $S^{\prime}=\{1,2,3\}$ then this portfolio has three direct children portfolios: $\{1,2\},\{1,3\}$ and $\{2,3\}$. 
If the profit function associated with a parent portfolio $S$ is concave, this is an important factor in determining the optimality of that portfolio. We can assess the concavity of the associated profit function simply by ensuring that all principal minors of $\gamma_{S}$ have positive determinants. As a result, if a product portfolio $S$ has an associated profit function which is concave, the the following theorem establishes a dominance relationship between parent and child portfolios.

Theorem 1: Assume $\left|\gamma_{S}\right|>0$ and $q_{S}$ is a positive optimal quantity vector for portfolio $S$ with concave profit function. Let $S^{\prime}$ be a product portfolio created by adding another product $j(j \notin S)$ into portfolio S. If $q_{S^{\prime}}$ is also a positive optimal quantity vector for portfolio $S^{\prime}$ and $\gamma_{S^{\prime}}$ is invertible, then

1. If $\left|\gamma_{S^{\prime}}\right|>0$, then $S^{\prime}$ dominates $S$; else

2. $S$ dominates $S^{\prime}$.

Proof. See Appendix D.

The key implication of this result is as follows. A parent portfolio $S^{\prime}$ dominates a child portfolio $S$ if and only if $S^{\prime}$ has an associated profit function which is concave and the quantity offerings for all products included in $S^{\prime}$ are all positive. In essence, this also implies that $S^{\prime}$ dominates all its child portfolios. This result can be used to reduce the the number of potential product portfolios which need to be evaluated so that the firm can identify an optimal portfolio of products.

We use the dominance relationship established through Theorem 1 to propose a search algorithm for finding the optimal product portfolio. More specifically, by starting with smaller children portfolios and adding single product variants to these portfolios, all potential portfolios for evaluation can be identified. The determinant of the average substitution matrix $\gamma$ for each children portfolio is the building block of each potential parent portfolios since these have already been evaluated and computed. Note that a portfolio with all product variants is unlikely to be optimal, especially if the substitution effects are high between the possible product variants. While the dominance result alone does not determine the optimal 
portfolio, it can be used as a basis to identify good candidate portfolios which can then be exhaustively evaluated to identify the optimal portfolio. The proposed algorithm to determine such an the optimal portfolio is as follows.

1. Set $i=0$ at iteration 0 . Maintain a list that contains a null portfolio $S=\{\emptyset\}$.

2. For each concave portfolio $S$ in the list, add another product $j$ such that $j \notin S$. Hence every $S \cup\{j\}$ is an $(i+1)$-product portfolio.

3. If, at iteration $i+1$, there is no $(i+1)$-product portfolio with concave profit function, go to Step 6; otherwise, continue to the next step.

4. For each $(i+1)$-product portfolio with concave profit function, examine the optimal quantities by solving the FOCs of the profit function. For each portfolio, if all quantities are positive, add this portfolio to a list of concave feasible parent portfolio and remove all children portfolios of this newly-added portfolio.

5. Set $i=i+1$, go to Step 2 .

6. Compare the profits of the portfolios in the list of concave feasible parent portfolio, and the optimal product portfolio is the one with highest profit.

Each iteration in the search algorithm saves computation time through two mechanisms. First, it is easy to check the concavity of the profit function corresponding to newly composed portfolios through an evaluation of the principal minors of the associated $\gamma$ matrix. Thus we save computation time by not proceeding to compute the quantity offerings of all the products in a portfolio through the FOC. Second, even if the objective function for a given portfolio is concave, it is possible that the quantity offerings for all products included in this portfolio are not positive. In this case again, we do not include such a portfolio as a possible one to evaluate for identifying the optimal portfolio. Note that when the optimal quantity value for a particular product variant is equal to zero in a parent portfolio, this scenario is equivalent to a direct child portfolio and has already been checked. 
The algorithm proposed above relies primarily on the result of Theorem 1 which establishes the dominance of a parent portfolio in relationship to a child portfolio. From a managerial perspective, this implies that identifying parent portfolios is quite important since this would allow the pruning of all child portfolios and hence, reduce the complexity of the problem being analyzed. Further, the notion that higher substitution effects play a role in identifying dominant portfolios is also useful since this could also reduce the search process and enable managers to focus on portfolios consisting of products with lower substitution effects.

A reasonable question following the results above concerns the specific properties of the optimal portfolio. Unfortunately, due to the complexity of the general model, more analytical results are difficult to characterize. In Section 4, we investigate a special case of FP design where a firm currently offering $n$ SPs would like to obtain insights as to whether to also design a single all-in-one FP and offer it to the market.

\section{AN ALL-IN-ONE MODEL}

Given this setting, the potential product set for the firm is $n$ SPs and one all-in-one fusion product $f$ that integrates all the functions of the $n$ SPs. This is obviously a special case of our general model and our focus is to gain insights into the composition of the optimal product portfolio. Thus, we primarily focus on assessing whether the FP is included in the optimal portfolio. To start with, since substitution only exists between each SP and the all-in-one, the substitution matrix $r$ is a relatively sparse $(n+1) \times(n+1)$ matrix as follows:

$$
r=\left[\begin{array}{ccccc}
1 & 0 & \cdots & 0 & r_{1, f} \\
0 & 1 & \cdots & 0 & r_{2, f} \\
\vdots & \vdots & \ddots & \vdots & \vdots \\
0 & 0 & \cdots & 1 & r_{n, f} \\
r_{f, 1} & r_{f, 2} & \cdots & r_{f, n} & 1
\end{array}\right]
$$


Based on this, the profit function for the firm is:

$$
\Pi_{A I O M}=\sum_{k=1}^{n} q_{k}\left(a_{k}-c_{k}-q_{k}-r_{k, f} q_{f}\right)+q_{f}\left(a_{f}-c_{f}-q_{f}-\sum_{k=1}^{n} r_{f, k} q_{k}\right)
$$

To start with, we define several product portfolios: (i) APS (All Product Strategy) which consists of all SPs and the FP (i.e., $A P S=\{1, \ldots, n, f\}$ ); (ii) NFPS (No Fusion Product Strategy) which consists of all SPs (i.e., NFPS $=\{1, \ldots, n\}$ ); (iii) SFPS (Single Fusion Product Strategy) which consists of only the fusion product (i.e., $S F P S=\{f\}$ ); and (iv) PFPS (Partial Fusion Product Strategy) which consists of some SPs and the fusion product.

For this special setting, the process to identify an optimal portfolio is relatively straight forward and we proceed as follows:

1. Evaluate portfolio $A P S$ as follows.

- Check whether the associated profit function for this portfolio is concave. This can be done by simply ensuring that $\left(1-\sum_{k=1}^{n} \gamma_{k, f}\right) \geq 0$. If this is not the case, then $A P S$ cannot be the optimal portfolio and goto 2; otherwise, continue.

- Compute $q_{f}=\frac{d_{f}-\sum_{k=1}^{n} \gamma_{k, f} d_{k}}{1-\sum_{k=1}^{n} \gamma_{k, f}}$ and $q_{k}=d_{k}-\gamma_{k, f} d_{f}($ for $k=1, \ldots, n)$.

- If $q_{f}$ and all $q_{k}$ are positive, then $A P S$ is the optimal portfolio and STOP else, goto 2 .

2. Evaluate portfolio NFPS. In this case, it is trivial to show that for this portfolio $q_{k}=d_{k} \forall k, q_{f}=0$, and $\Pi=\sum_{i=1}^{n} d_{k}^{2}$.

3. Evaluate portfolio SFPS. In this case, it is trivial to show that for this portfolio $q_{f}=d_{f}, q_{k}=0 \forall k$, and $\Pi=d_{f}^{2}$.

4. Evaluate all possible portfolios PFPS. Using the dominance relationship established through Theorem 1, compare all possible parent portfolios in this set to identify the "best" PFPS (defined as one which provides the maximum profit). If such a portfolio exists, goto step 6, else goto step 5 . 
5. Compare portfolios NFPS and SFPS and the one with the higher profit is optimal and Stop.

6. Compare the "best" PFPS portfolio in step 4 to the NFPS portfolio and the one with the higher profit is optimal.

As is obvious, Step 4 in this process is computationally intensive. However, given the sparsity of $\gamma$, it is much easier to implement the process to search among all PF PS portfolios. Recall that each potential PFPS portfolio contains some combination of the SPs and the fusion product $f$. Define $K$ as the set of SPs included in a specific PFPS - call this portfolio PFPS $S_{K}$. For this portfolio, define $\lambda_{K}=\sum_{k \in K} d_{k} \gamma_{k, f}$ and $\theta_{K}=1-\sum_{k \in K} \gamma_{k, f}^{2}$. Then if $\theta_{K} \geq 0$, the profit function associated with portfolio $P F P S_{K}$ is concave. Further, the optimal quantity offerings for all products included in portfolio $P F P S_{K}$, can easily be computed as $q_{f}=\frac{d_{f}-\lambda_{K}}{\theta_{K}}$ and $q_{k}=d_{k}-\gamma_{k, f} q_{f}$ for all $k \in K$. Of course, if the profit function is concave and all these quantity offerings are positive, then $P F P S_{K}$ is a candidate portfolio for evaluation in step 4. In terms of the resulting profit for $P F P S_{K}$, this can also be determined quite easily as $\Pi_{P F P S_{K}}=\sum_{k \in K} d_{k}^{2}+\theta_{K}{ }^{-1}\left(d_{f}-\lambda_{K}\right)^{2}$.

In the final steps of the process outlined above, steps 5 and 6 require some explanation. Note that if there exists even one PFPS which is a candidate for an optimal solution, then according to the process described above, portfolio SFPS is never in contention as an optimal portfolio since SFPS is always a "child" portfolio for any potential PFPS. This justifies skipping step 5 provided there is at least one PFPS which is identified as a candidate in step 4. It follows that the comparison in Step 6 (between the "best" PFPS and NFPS) is also quite straightforward. Assume that $K^{*}$ represents the set of SPs in the 'best' PFPS identified in step 4. Then if $\theta_{K^{*}}^{-1}\left(d_{f}-\lambda_{K^{*}}\right)^{2}>\sum_{k \notin K^{*}} d_{k}^{2}$, PFPS dominates NFPS and vice versa. Of course, if there is no PFPS which is feasible (which is quite unlikely), step 5 simplifies the search process for the optimal portfolio by simply comparing portfolios NFPS and SFPS.

As a direct result of this algorithm, managers can more easily determine the optimal 
fusion product portfolio. In summary, if the all product strategy is concave and all quantities are positive, then APS is the optimal portfolio; otherwise, complement the fusion product with any subset of single-function products to evaluate whether any PFPS can be concave and feasible and find the best PFPS. If any PFPS exists, compare the best PFPS with the NFPS that contains all single-function products; else, compare the NFPS with the SFPS, which includes only the fusion product.

The results for this special setting (where a firm can offer a single FP incorporating the functionalities of $n$ distinct SPs) indicate that it is highly likely that the FP will be included in the firm's optimal product portfolio (since it is included in $S F P S, A P S$, and all possible PF PS). From an FP design perspective, this implies that a firm should make an attempt to design an FP which integrates the $n$ functionalities included in each SP. In the next section, we focus on the general case where the firm can design FPs with any combination of $n$ functions. Given that this problem is analytically complex, we resort to a numerical analysis based on secondary data.

\section{NUMERICAL EXAMPLES}

The analysis in Section 4 is related to the product design decision when a firm can offer an all-in-one device which integrates $n$ functions together. Since the firm has the technology to fuse $n$ SPs together, it is likely that technology is available to fuse subsets of the component products. However, because of the complexity of this more general problem, analytic results are difficult to obtain. In this section, we perform numerical analysis to gain further insight into this problem. Specifically, the dynamics of changes in the substitution indices and the profit margins are investigated for the complete model as discussed in Section 3.

For the numerical examples, we use Sony to illustrate a firm who offers fusion products based on digital camera, MP3 music player, and cell phone functionalities. Sony is a manufacturer of digital cameras and MP3 players, and it also maintains a joint-venture with Ericsson to produce cell phones, which adopts Sony's technology to provide multi-function 
cell phones (Bryan-low, 2007). Sony owns 50\% share of Sony Ericsson (SE hereafter); hence, Sony has strong influence in the joint-venture's strategy. Moreover, recent articles have discussed how SE has adjusted the number of product variants that it is providing to the market (Regan, 2007). The president of the corporation has commented that, "We are confident that the remainder of the year will see us further capitalize on this new broader portfolio," which includes cell phone, camera and MP3 product variants.

We index the cell phone, the digital camera, and the MP3 player as (single-function) products 1, 2, and 3, respectively. In terms of SPs, product models SE T105, CyberShot, DSC-S700, and Walkman NWZ-A816 are examples of products 1, 2, and 3, respectively. Since all cell phones offered by $S E$ in 2007 have extra functions, we use an older model (T105) as an example of a single function cell phone. Products 4, 5, and 6 are some-inone products which combine two of the single-function products together. $S E$ K550i is an example of product 4 that integrates functions of digital camera and cell phone. Sony does not provide products 5 and 6 to the market that combine a cell phone and an MP3 player or a digital camera with an MP3 player. For the three-function all-in-one product, SE W810i is an example of product 7 in our model. Note that since the camera phones normally adopt low image resolution, we choose a low-end digital camera model and only analyze the impact of fusion products on the low-end market.

Regarding profit margin estimations, we use data from Sony and $S E$ 's annual report. The average gross profit margin rate of $S E$ in the last three year is $28 \%$, while Sony's annual report shows that the company-wide gross profit margin is 37\%. There is no available profit margin data from any of Sony's specific product categories. Therefore, we utilize industry data from Sony's competitors to estimate the gross profit margins for the SPs. According to news reports, Nokia's cell phone (Williams, 2005), Canon's digital camera (Rowley, 2007), and Creative's MP3 player have gross profit margins at $15 \%, 23 \%$ and $23 \%$, respectively. Based on the market price data, the prices of products $1,2,3,4$, and 7 are $\$ 120, \$ 150$, $\$ 150, \$ 200$ and $\$ 240$. We extrapolate the prices for products 5 and 6 , since these are not 
currently offered by Sony. The profit margin is then calculated using both the unit price and the gross profit margin. According to this estimation method, the scaled profit margins $\left(d_{k}\right)$ of products 1 to 7 used in our analysis are set as $\$ 24, \$ 38, \$ 38, \$ 50, \$ 53, \$ 57$, and $\$ 67$, respectively.

To characterize the impact of the substitution matrix on the optimal solution, we actually consider two different sample matrices $\mathrm{A}$ and $\mathrm{B}$ as shown in Table 2 and Table 3 . These two matrices allow us to capture some effects of the landscape of substitutability indices on the optimal solutions. The values shown in matrix A are more realistic for Sony's three product market, in that there are relatively high substitution indices between the products which contain similar functions. These single-function products under consideration are fairly congruent, in that it is easy to fuse them into a single product and the newly fused product is serving a similar market as the original single-function products. In contrast, the values shown in matrix B reflect those associated with a more incongruent set of products. The substitution indices are lower, as the combination products seem to create a new market with less overlap with the original markets for the single-function products.

Insert Tables 2 and 3 here

We consider symmetric substitution matrices for the seven products as shown in Tables 2 and 3. Note that this analysis also applies to any asymmetric substitution matrices which can be 'averaged' to find these two matrices. A zero in the matrix denotes the fact that there are no substitution effects for the corresponding product pair. For example, because products 1, 2, and 3 are single-function products, there is no functional overlap between these different markets. Consequently, the values for $\gamma_{i, j}^{A}$ and $\gamma_{i, j}^{B}$ between these three products are equal to zero.

The results of six different numerical examples are shown in Table 4. A summary of the input parameters as well as the corresponding optimal portfolio and objective values are shown for each example. The first three cases correspond to the cell-phone, digital camera, and MP3 player markets and use matrix A for the substitution matrix. Case 1 reflects the 
initial scenario and in this case, an all-in-one FP should be designed and offered to the market. Cases 2 and 3 show the impact of changes in specific profit margin parameters on the optimal portfolio. For case 2, an increase in the profit margin for the the cell phone (product 1) has no effect on the product offering. Hence, in both cases 1 and 2, the the all-in-one FP should be designed and then offered to the market.

For case 3, an increase in the profit margin for the third single-function product (i.e. the MP3 player) changes the optimal portfolio slightly. In this case, it is now optimal to offer the single-function digital camera, single-function MP3 player and two-function camera phone to the market. This result is supported in the press by anecdotal evidence which points to the popularity of camera phone. The research report released by ABI Research (2005) projects that the shipment of camera phones is predicted to surpass the shipment of single-function cell phones. These industry trends provide face validity to the results of our model since the design of a single device integrating the functionalities of a high-end digital camera and the cell phone is an optimal strategy.

Insert Table 4 here

The remaining examples utilize the matrix $\mathrm{B}$ which reflects a more incongruent product set with lower substitution indices. In general, the optimal product portfolio for these examples includes more FP variants and is more sensitive to parameter changes than those shown for matrix A. In comparing case 1 to case 4, the optimal product portfolio includes both the all-in-one fusion product (i.e. product 7) and also a some-in-one product (i.e. product 4). Specifically, those customers in the market for only the first or second singlefunction products (i.e. those contained in product 1 or product 2) will choose between the two different products (i.e. product 4 or product 7). Customers in the market for the third single-function product (i.e. product 3) will buy the all-in-one fusion product (i.e. product 7). In this case, the optimal strategies are to design an all-in-one device and a camera phone.

In cases 5 and 6, the profit margin parameters are varied for SPs 2 and 3 and the optimal product portfolio for these cases changes significantly. In case 5, the profit margin 
for product 2 is increased. As a consequence, the optimal product portfolio now includes the single-function product 2 and the some-in-one product 5, which indicates the firm should design and offer digital camera (for function 2) and MP3 phone (for functions 1 and 3) to the aggregate market. Similarly, in case 6, the profit margin for product 3 is increased. Consequently, the optimal product portfolio now includes all single-function products 1, 2 and 3, and the some-in-one product 4 . Cases 5 and 6 imply that, ceteris paribus, the allin-one FP should not be designed and offered as one of the SPs becomes more profitable. This may happen when adding three functions significantly decreases the usability such that there is a lack of synergy between the single-function products. Interestingly, the result from these cases implies that when a single function product is associated with a relatively high profit margin, the firm should not combine this function with others to sell it as part of a fusion product.

Some additional managerial insights based on this analysis are as follows. First, when the substitution effects are relatively high, a portfolio containing a smaller number of products is likely to be optimal. If a single all-in-one fusion product has high margins, then this product dominates the product portfolio. However, when a stand alone single function product has relatively high profit margins, then it is less likely that a fusion product containing this function should be offered. Instead, the firm should design and offer the single function product independently and combine other lower margin functions into a some-in-one product. Lastly, small changes in parameter values can cause large changes in the optimal portfolio. When the set of products under consideration is somewhat incongruent (i.e. the substitution effects are low), then the product portfolio in general is somewhat larger and more sensitive to small changes in the profit margins.

\section{CONCLUSION AND FUTURE EXTENSIONS}

If technology makes it possible to integrate many functions into one device, firms might be contemplating introducing fusion products into the market. However, this may lead to 
product proliferation, excessive self cannibalization, and consumer "feature fatigue." Manufacturers must decide how to intelligently fuse these technologies into different product variants so as to design an appropriate fusion product for the market. We have analyzed a normative model to gain insights into this design decision. Even though the number of possible product portfolio configurations is large, we develop an algorithm which can exploit parent-child portfolio relationships with a simple check on concavity properties of the objective function.

There are several managerial insights generated from the analysis of the model. In general, the optimal portfolio and hence the 'best' product designs are a function of two important parameters: profit margin and substitution effects. A product with higher profit margin and smaller substitution effects with other products is more likely to be included in an optimal portfolio and thus, this product design should be initiated. However, facing any possible combination of function integrations, substitution and cannibalization cannot be avoided if the firm intends to offer many different products. Since the firm's objective is profit maximization, a careful investigation and evaluation of all (single-function, somein-one, all-in-one) possible products is the best way to achieve optimality while taking into account the cannibalization effects.

In general, the firm should not manufacture too many different fusion products (FPs) simultaneously. Strong cannibalization effects among these FPs imply selecting the right fusion product is important. In essence, the firm should not complement the FP with too many component or other FPs when the substitutability indices are high. It is also interesting to note that our general-form model can also be applied to the problem of product variety in a certain market segment. Kraft Foods, Inc. (Ellison, 2003) found they have launched too many similar products in one market segment. Introducing too many products induces strong cannibalization among their own products.

In contrast, the product portfolio and corresponding product design is more difficult to determine when cannibalization effects are small. This situation can occur when the set 
of single-function products (SPs) under consideration are incongruent, or when the fusion products (FPs) create a significantly different market than the original SPs. Numerical results show that the optimal portfolio in this situation generally contains a wider variety of products and is more sensitive to changes in the profit and cannibalization parameters.

In summary, the managerial insights pertaining to the fusion product portfolio problem follow:

1. If a single all-in-one fusion product has relatively high margins, then this product likely dominates the product portfolio.

2. When a stand alone single function product has relatively high profit margins, then it is less likely that a fusion product containing this product should be offered. In this case, the firm should design and offer the single function product independently and combine other lower margin functions into a some-in-one product.

3. Similar to other combinatorial problems, small changes in parameter values can cause large changes in the optimal product portfolio.

4. When substitution effects are relatively high (i.e. the product set is more congruent), a portfolio containing a smaller number of products is more likely to be optimal.

5. When substitution effects are relatively low (i.e. the product set is more incongruent), then the optimal product portfolio is generally larger in size and more sensitive to small changes in profit margins.

Of course, there are limitations associated with analytical models of this type which should be addressed in future work (Loch, Pich, Terwiesch, \& Urbschat, 2001). While this model offers a strategic level tool incorporating market level and substitution effects, other factors influencing system level design and detail design configurations should be considered.

Several future extensions of this model warrant further investigation. While we assume that the technology already exists which enables a firm to produce a fusion product, an 
alternative model could help managers to determine which technology investments would be most beneficial. Other issues typically associated with product design should also be considered. When the manufacturer tries to fuse many functions into an all-in-one device, the product space, platform and human interface are confined in a limited scale. How should the manufacturer design the fusion product? Which function should the fusion product use as the platform base? When the fusion product's dimensions are physically too small to put all functions together, how should a firm segment different FPs? While we present a single firm model, the impact of the competitive nature of fusion product markets is an important topic. Finally, an empirical examination of new product introduction strategies analyzed in this paper would be a natural extension of this research. 


\section{REFERENCES}

ABI Research. 2005. Camera Phones to Steal Low-End Digital Camera Market within Two Years. August 10.

Adams, W. J., \& J. L. Yellen. 1976. Commodity Bundling and the Burden of Monopoly. The Quarterly Journal of Economics 90(3), 475-498.

Allenby, G., \& P. E. Rossi. 1991. Quality Perceptions and Asymmetric Switching between Brands. Marketing Science 10(3), 185-204.

Anonymous. 1997. A Fax Machine or More? Consumer Reports 62(9), 32.

Anonymous. 2006. Nokia Predicts Lower Profit Margins in Coming Years. http://www . teleclick.ca/2006/11/nokia-predicts-lower-profit-margins-in-coming-years/.

Ansari, A., N. Economides, \& A. Ghosh. 1994. Competitive Positioning in Markets with Nonuniform Preferences. Marketing Science 13(3), 248-273.

Avery, S. 2004. MFP Demand Strengthens: HP Enters Crowded Market. Purchasing 133(1), 48.

Aydin, G., \& J. K. Ryan. 2000. Product Line Selection and Pricing Under the Multinomial Logit Choice Model. Working Paper, Purdue University.

Bakos, Y., \& E. Brynjolfsson. 2000. Bundling and Competition on the Internet. Marketing Science 19(1), 63-82.

Baumol, W. J. 1967. Calculation of Optimal Product and Retailer Characteristics: The Abstract Product Approach. Journal of Political Economy 75(5), 674-685.

Ben-Akiva, M., \& S. Gershenfeld. 1998. Multi-Featured Products And Services: Analysing Pricing And Bundling Strategies. Journal of Forecasting Volume 17(3-4), 175-196.

Bryan-low, C. 2007. Sony Ericsson's Basic-Cellphone Plan. Wall Street Journal. August 30, B3.

Burgelman, R., C. Christensen, \& S. Wheelwright, 2003. Strategic Management of Technol- 
ogy and Innovation, McGraw Hill/Irwin, Boston.

Cachon, G. P., C. Terwiesch, \& Y. Xu. 2005. Retail Assortment Planning in the Presence of Consumer Search. MSOM 7, 330-346.

Chen, K. D., \& W. H. Hausman. 2000. Mathematical Properties of the Optimal Product Line Selection Problem Using Choice-Based Conjoint Analysis. Management Science $46(2), 327-332$.

Chen, Y., A. J. Vakharia, \& A. Alptekinoğlu. 2008. Product Portfolio Strategies: The Case of Multi-Function Products. Production \& Operations Management 17(6), 587-598.

Dansby, R. E., \& C. Conrad. 1984. Commodity Bundling. The American Economic Review $74(2), 377-381$.

Dasci, A., \& G. Laporte. 2004. Location and Pricing Decisions of A Multistore Monopoly in A Spatial Market. Journal of Regional Science 44(3), 489-515.

Deneckere, R. J., \& A. de Palma. 1998. The Diffusion of Consumer Durables in a Vertically Differentiated Oligopoly. The RAND Journal of Economics 29(4), 750-771.

Dobson G., \& S. Kalish. 1988. Positioning and Pricing A Product Line. Marketing Science $7(2), 107-125$.

Dobson, G., \& C. A. Yano. 2002. Product Offering, Pricing, and Make-to-Stock / Make-toOrder Decisions with Shared Capacity. Production and Operations Management 11(3), 293-312.

Ellison, S. 2003. Krafts Stale Strategy; Endless Extensions of Oreos, Chips Ahoy and Jello-O Brands Created a New-Product Void. Wall Street Journal. December 18, B1.

Fusfeld, A. 1978. "How to put technology into corporate planning," Technology Management Review 80(6) 51.

Gabszewicz, J. J., \& J.-F. Thisse. 1979. Price Competition, Quality and Income Disparities. Journal of Economics Theories 20, 340-359. 
Hanson, W., \& R. K. Martin. 1990. Optimal Bundle Pricing. Management Science 36(2), $155-174$.

Harbaugh, L. 1998. Find the Right Balance When Combining Functions. Information Week. Jun. 29, 120 .

Hendel, I. 1999. Estimating Multiple-Discrete Choice Models: An Application to Computerization Returns. Review of Economic Studies 66(2), 423-446.

Hopp, W. J., \& X. Xu. 2005. Product Line Selection and Pricing with Modularity in Design. Manufacturing \& Service Operations Management 7(3), 172-187

Jacobi, C. G. 1833. De formatione et proprietatibus Determinantium. Crelle's Journal xxii, 285-318.

Kaicker, A., W. O. Bearden, \& K. C. Manning. 1995. Component versus Bundle Pricing: The Role of Selling Price Deviations from Price Expectations. Journal of Business Research 33(3), 231-239.

Kaul, A., \& V. R. Rao. 1995. Research for Product Positioning and Design Decisions: An Integrative Review. International Journal of Research in Marketing 12(4), 293-320.

Lancaster, K. 1990. The Economics of Product Variety: A Survey. Marketing Science 9(3), 189-206.

Li, L., \& H. Zhang. 2001. Supply Chain Information Sharing in a Competitive Environment. In J.-S. Song, \& D. D. Yao, eds. Supply Chain Structures: Coordination, Information and Optimization Kluwer Academic Publishers, Boston, MA.

Loch, C. H., M. T. Pich, C. Terwiesch, \& M. Urbschat. 2001. "Selecting R\&D Projects at BMW: A Case Study of Adopting Mathematical Programming Models IEEE Transactions on Engineering Management, 48(1), 70-80.

Loch, C. H., \& S. Kavadias. 2002. Dynamic Portfolio Selection of NPD Programs Using Marginal Returns. Management Science 48(10), 1227-1241. 
Magid, L. J. 1998. The Cutting Edge / Personal Technology; All-in-One Devices Improve. Los Angeles Times April 20, 5.

Mahajan S., \& G. van Ryzin. 2001. Stocking Retail Assortments under Dynamic Consumer Substitution. Operations Research 49(3), 334-351.

McAfee, R. P., J. McMillan, \& M. D. Whinston. 1989. Multiproduct Monopoly, Commodity Bundling, and Correlation of Values. Quarterly Journal of Economics 104(2), 371-383.

Meyers, J. 2004. Game Theory, Teen-Style. American Demographics 24(4), 10.

Moorthy, K. S. 1984. Market Segmentation, Self-Selection, and Product Line Design. Marketing Science 3(4), 288-307.

Moorthy, K. S., \& I. P. L. Png. 1992. Market Segmentation, Cannibalization, and the Timing of Product Introductions. Management Science 38(3), 345-359.

Mulhern, F. J., \& R. P. Leone. 1991. Implicit Price Bundling of Retail Products: A Multiproduct Approach to Maximizing Store Profitability. Journal of Marketing 55(4), 63-76.

Pekgun, P., P. M. Griffin, \& P. Keskinocak. 2005. Coordination of Marketing and Production for Price and Leadtime Decisions. Working Paper, Georgia Institute of Technology.

Ramdas, K., \& M. S. Sawhney. 2001. Cross-Functional Approach to Evaluating Multiple Line Extensions for Assembled Products. Management Science 47(1), 22-36

Regan, K. 2007. Move to Cheaper Handsets Pares Sony Ericsson Profit. E-Commerce Times. October 11, http://www. ecommercetimes.com/story/59771.html.

Rowley, I. 2007. Japan's Digital Camera Picture Still Bright. Businessweek.com/. April 10, http://www. businessweek.com/print/globalbiz/content/apr2007/gb20070410_781215. htm.

Rust, R. T., D. V. Thompson, \& R. W. Hamilton. 2006. Defeating Feature Fatigue. Harvard Business Review 84(2), 98-107. 
Rysavy, P. 2004. Device Diversity. Network Computing 15(7), 36.

Schmalensee, R. 1982. Commodity Bundling by Single-Product Monopolies. Journal of Law and Economics 25(1), 67-71.

Schmalensee, R. 1984. Gaussian Demand and Commodity Bundling. Journal of Business $57(1), 211-230$.

Schonfeld, E. 2004. BlackBerry Season Riding a Huge Comeback, Research in Motion Finds Itself Surrounded by Rivals, All Gunning for A Share of the Booming Wireless Market It Created.Business 2.0 5(9), 132-140.

Sethuraman, R., V. Srinivasan, \& D. Kim. 1999. Asymmetric and Neighborhood Cross-Price Effects: Some Empirical Generalizations. Marketing Science 18(1), 23-41.

Simonin, B. L., \& J. A. Ruth. 1995. Bundling As A Strategy for New Product Introduction: Effects on Consumers' Reservation Prices for the Bundle, the New Product, and Its Tie-in. Journal of Business Research 33(3), 219-230.

Singh, N., \& X. Vives. 1984. Price and Quantity Competition in A Differentiated Duopoly. RAND Journal of Economics 15(4), 546-554.

Smith, S. A., \& N. Agrawal. 2000. Management of Multi-Item Retail Inventory Systems with Demand Substitution Operations Research 48(1), 50-64.

Sorenson, O. 2000. Letting the Market Work for You: An Evolutionary Perspective on Product Strategy. Strategic Management Journal 21(5), 577-592.

Stigler, G. J. 1968. A Note on block Booking. In G. J. Stigler, ed. The Organization of Industry Richard D. Irwin, Inc. Homewood, IL.

Thompson, D. V., R. W. Hamilton, \& R. T. Rust. 2005. Feature Fatigue: When Product Capabilities Become Too Much of a Good Thing. Journal of Marketing Research 42(4), $431-42$.

Ulrich, K. T., S. D. Eppinger. 2000. Product Design and Development, McGraw Hill/Irwin, 
Boston, 14-31.

Vandenbosch, M. B., \& C. B. Weinberg. 1995. Product and Price Competition in a TwoDimensional Vertical Differentiation Model. Marketing Science 14(2), 224-249.

van Mieghem, J. A., \& M. Dada. 1999. Price versus Production Postponement: Capacity and Competition. Management Science 45(12), 1631-1649.

van Ryzin, G., \& S. Mahajan. 1999. On the Relationship between Inventory Costs and Variety Benefits in Retail Assortments. Management Science 45(11), 1496-1509.

Walker, R. 2004. A Status Gadget Implies Almost Limitless Functionality and Practicality. New York Times Magazine January 25, 22.

Wauthy, X. 1996. Quality Choice in Models of Vertical Differentiation. Journal of Industrial Economics 44(3), 345-353.

Williams, M. 2005. MP3 player sales drive Creative to record Q3. The Standard, April 22, http://www. thestandard.com/internetnews/002990.php.

Yadav, M. S., \& K. B. Monroe. 1993. How Buyers Perceive Savings in a Bundle Price: An Examination of a Bundle's Transaction Value. Journal of Marketing Research 30(3), 350-358. 


\section{APPENDIX A}

Taking the first and second derivatives of the objective function for problem (GP), we obtain

$$
\begin{aligned}
\frac{\partial \Pi}{\partial q_{k}} & =2 d_{k}-2 q_{k}-\sum_{j=1, j \neq k}^{m}\left(r_{k, j}+r_{j, k}\right) q_{j}, \quad k=1,2, \ldots, m, \\
\frac{\partial^{2} \Pi}{\partial q_{k}^{2}} & =-2 \quad k=1,2, \ldots, m \\
\frac{\partial^{2} \Pi}{\partial q_{k} \partial q_{j}} & =-\left(r_{k, j}+r_{j, k}\right), \quad k, j=1,2, \ldots, m, k \neq j .
\end{aligned}
$$

Based on this:

$$
\begin{aligned}
H & =\left[\begin{array}{ccccc}
-2 & -\left(r_{1,2}+r_{2,1}\right) & -\left(r_{1,3}+r_{3,1}\right) & \cdots & -\left(r_{1, m}+r_{m, 1}\right) \\
-\left(r_{1,2}+r_{2,1}\right) & -2 & -\left(r_{2,3}+r_{3,2}\right) & \cdots & -\left(r_{2, m}+r_{m, 2}\right) \\
-\left(r_{1,3}+r_{3,1}\right) & -\left(r_{2,3}+r_{3,2}\right) & -2 & \cdots & -\left(r_{3, m}+r_{m, 3}\right) \\
\vdots & \vdots & \ddots & \vdots & \vdots \\
-\left(r_{1, m}+r_{m, 1}\right) & -\left(r_{2, m}+r_{m, 2}\right) & -\left(r_{3, m}+r_{m, 3}\right) & \cdots & -2
\end{array}\right] \\
& =(-2)\left[\begin{array}{ccccc}
1 & \gamma_{1,2} & \gamma_{1,3} & \cdots & \gamma_{1, m} \\
\gamma_{1,2} & 1 & \gamma_{2,3} & \cdots & \gamma_{2, m} \\
\gamma_{1,3} & \gamma_{2,3} & 1 & \cdots & \gamma_{3, m} \\
\vdots & \vdots & \ddots & \vdots & \vdots \\
\gamma_{1, m} & \gamma_{2, m} & \gamma_{3, m} & \cdots & 1
\end{array}\right]=(-2) \gamma,
\end{aligned}
$$

where $\gamma_{k, j}=\frac{1}{2}\left(r_{k, j}+r_{j, k}\right)(k, j \in\{1,2, \ldots, n\}$ and $k \neq j)$ represents the average substitution effect between products $k$ and $j$. 


\section{APPENDIX B}

The equation system for the FOCs is shown below. Solving the optimal quantity vector by Cramer's rule, we obtain the following:

$$
\begin{aligned}
& \gamma^{T} q=\left[\begin{array}{ccccc}
1 & \gamma_{1,2} & \gamma_{1,3} & \cdots & \gamma_{1, m} \\
\gamma_{1,2} & 1 & \gamma_{2,3} & \cdots & \gamma_{2, m} \\
\gamma_{1,3} & \gamma_{2,3} & 1 & \cdots & \gamma_{3, m} \\
\vdots & \vdots & \ddots & \vdots & \vdots \\
\gamma_{1, m} & \gamma_{2, m} & \gamma_{3, m} & \cdots & 1
\end{array}\right]\left[\begin{array}{c}
q_{1} \\
q_{2} \\
q_{3} \\
\vdots \\
q_{m}
\end{array}\right]=\left[\begin{array}{c}
\left(a_{1}-c_{1}+\nu_{1}\right) / 2 \\
\left(a_{2}-c_{2}+\nu_{2}\right) / 2 \\
\left(a_{3}-c_{3}+\nu_{3}\right) / 2 \\
\vdots \\
\left(a_{m}-c_{m}+\nu_{m}\right) / 2
\end{array}\right] \\
& =\left[\begin{array}{c}
d_{1}+\frac{\nu_{1}}{2} \\
d_{2}+\frac{\nu_{2}}{2} \\
d_{3}+\frac{\nu_{3}}{2} \\
\vdots \\
d_{m}+\frac{\nu_{m}}{2}
\end{array}\right]=d+\frac{\nu}{2} \\
& q^{*}=\left[\gamma^{T}\right]^{-1}\left(d+\frac{1}{2} \nu\right)=[\gamma]^{-1}\left(d+\frac{1}{2} \nu\right) \\
& \nu \geq 0 \\
& q_{k} \nu_{k}=0, \forall k
\end{aligned}
$$




\section{APPENDIX C}

Let $S$ be any portfolio with all positive-quantity products, that is $q_{k}>0, \forall k \in S$, then the optimal quantities and the profit are shown in 9 and 10, respectively.

$$
\begin{aligned}
& q_{S}=\left[\gamma_{S}^{T}\right]^{-1} d_{S}=\left[\gamma_{S}\right]^{-1} d_{S} \\
\Pi_{S}= & q_{S}^{T}\left(p_{S}-c_{S}\right)=q_{S}^{T}\left(2 d_{S}-r_{S} q_{S}\right)=2 q_{S}^{T} d_{S}-q_{S}^{T} r_{S} q_{S} \\
= & \frac{2 d_{S}^{T} \operatorname{Adj}\left[\gamma_{S}\right] d_{S}}{\left|\gamma_{S}\right|}-\frac{d_{S}^{T}\left[\gamma_{S}\right]^{-1} r_{S} A d j\left[\gamma_{S}\right] d_{S}}{\left|\gamma_{S}\right|} \\
= & \frac{d_{S}^{T}\left\{2 I_{s}-\left[\gamma_{S}\right]^{-1} r_{S}\right\} \operatorname{Adj}\left[\gamma_{S}\right] d_{S}}{\left|\gamma_{S}\right|} \\
= & \frac{d_{S}^{T}\left\{2 I_{s}-\left[\gamma_{S}\right]^{-1}\left[2 \gamma_{S}-r_{S}^{T}\right]\right\} A d j\left[\gamma_{S}\right] d_{S}}{\left|\gamma_{S}\right|} \\
= & \frac{\left.d_{S}^{T}\left\{2 I_{s}-2\left[\gamma_{S}\right]^{-1} \gamma_{S}+\left[\gamma_{S}\right]^{-1} r_{S}^{T}\right]\right\} A d j\left[\gamma_{S}\right] d_{S}}{|\gamma|} \\
= & d_{S}^{T}\left[\gamma_{S}\right]^{-1} r_{S}^{T}\left[\gamma_{S}\right]^{-1} d_{S}=\left[\left[\gamma_{S}\right]^{-1} d_{S}\right]^{T} r_{S}^{T}\left[\gamma_{S}\right]^{-1} d_{S} \\
= & q_{S}^{T} r_{S}^{T} q_{S}
\end{aligned}
$$




\section{APPENDIX D}

\section{Proof for Theorem 3.3}

The profit difference between the parent $S^{\prime}$ and the child $S$ portfolios is

$$
\begin{aligned}
\Pi_{S^{\prime}}-\Pi_{S} & =\frac{\left|\gamma_{S}\right| d_{S^{\prime}}^{T} \operatorname{Adj}\left[\gamma_{S^{\prime}}\right] d_{S^{\prime}}-\left|\gamma_{S^{\prime}}\right| d_{S}^{T} \operatorname{Adj}\left[\gamma_{S}\right] d_{S}}{\left|\gamma_{S}\right|\left|\gamma_{S^{\prime}}\right|} \\
& =\frac{\left|\gamma_{S^{\prime}}\right|}{\left|\gamma_{S}\right|}\left(q_{S_{j}^{\prime}}\right)^{2},
\end{aligned}
$$

where the last equality follows from Corollary 1 in Appendix E. Because $\left|\gamma_{S}\right|>0$, we obtain

$$
\Pi_{S^{\prime}}-\Pi_{S} \begin{cases}>0, & \text { if }\left|\gamma_{S^{\prime}}\right|>0 \\ <0, & \text { if }\left|\gamma_{S^{\prime}}\right|<0\end{cases}
$$




\section{APPENDIX E}

We shall state and prove a general theorem which may be have broader application than the special case of this paper. For this reason, we will treat the general result using different notation.

Let $A$ be an $(n \times n)$ matrix and let $B$ be the $(n-1) \times(n-1)$ submatrix obtained by deleting the last row and last column of $A$. Let $\operatorname{Adj}(A)$ and $\operatorname{Adj}(B)$ be the adjugate matrices of $A$ and $B$. Let $\mathbf{x}^{\prime}=\left(x_{1}, \ldots, x_{n}\right)^{T}, \mathbf{x}=\left(x_{1}, \ldots, x_{n-1}\right)^{T}, \mathbf{y}^{\prime}=\left(y_{1}, \ldots, y_{n}\right)^{T}$ and $\mathbf{y}=\left(x_{1}, \ldots, y_{n-1}\right)^{T}$.

Theorem 2. Let $R$ be the matrix obtained from $A$ by replacing the last row by $\mathrm{x}^{\prime T}$ and let $C$ be the matrix obtained from $A$ by replacing the last column by $\mathbf{y}^{\prime}$. Then

$$
|B| \mathbf{x}^{\prime T} \operatorname{Adj}(A) \mathbf{y}^{\prime}-|A| \mathbf{x}^{T} \operatorname{Adj}(B) \mathbf{y}=|R||C|
$$

Proof. First, we recall the standard notation $A_{i, j}$ for the the $(n-1) \times(n-1)$ submatrix obtained from $A$ by deleting row $i$ and column $j$. Also, the $(i, j)$ cofactor of $A$ is $(-1)^{i+j}\left|A_{i, j}\right|$. Then $\operatorname{Adj}(A)$ is the matrix whose $(i, j)$ entry is the $(j, i)$ cofactor of $A$. To prove Theorem 2, we must reformulate it a little. Let $(\operatorname{Adj}(B))^{+}$be the $n \times n$ matrix obtained from $\operatorname{Adj}(B)$ by adding a last row and column of zeros and let $\mathrm{F}$ be defined as:

$$
F:=|B| \operatorname{Adj}(A)-|A|(\operatorname{Adj}(B))^{+}
$$

Then equation (11) is equivalent to

$$
\mathbf{x}^{\prime T} F \mathbf{y}^{\prime}=|R \| C|
$$

Let $\mathbf{r}$ be the column vector whose $i$-th entry is the $(n, i)$ cofactor $(-1)^{i+n}\left|A_{n, i}\right|$ of $A$. Similarly, let $\mathbf{c}$ be the column vector whose $i$-th entry is the $(i, n)$ cofactor of $A$. (Thus $\mathbf{r}=\mathbf{c}$ if $A=A^{T}$.) 
Then by the cofactor expansion of determinants, we have

$$
|R|=\mathbf{x}^{\prime T} \mathbf{r} \quad \text { and } \quad|C|=\mathbf{c}^{T} \mathbf{y}^{\prime}
$$

so we can rewrite $(12)$ as

$$
\mathbf{x}^{\prime T} F \mathbf{y}^{\prime}=\mathbf{x}^{\prime T} \mathbf{r c}^{T} \mathbf{y}^{\prime}
$$

This equation expresses the equality of two bilinear forms. Since two bilinear forms are equal if and only if they are represented by the same matrices, Theorem 2 is equivalent to

$$
F=\mathbf{r c}^{T}
$$

For $1 \leq i, j \leq n$, let $f_{i j}$ denote the $(i, j)$ entry of $F$. Then (14) is equivalent to the equations

$$
f_{i j}=(-1)^{i+j}\left|A_{n, i}\right|\left|A_{j, n}\right|, \quad(1 \leq i, j \leq n) .
$$

To prove (15) we now examine each entry of $F$, using the definition (11). If either the row index or the column index is equal to $n$, then the entry is simply that of $|B| \operatorname{Adj}(A)$. Thus,

$$
\begin{aligned}
f_{n n} & =\left|A_{n, n}\right||B|, \quad \text { and for } 1 \leq i \leq n-1, \\
f_{i n} & =(-1)^{i+n}\left|A_{n, i}\right||B|, \\
f_{n i} & =(-1)^{n+i}\left|A_{i, n}\right||B| .
\end{aligned}
$$

Since $B=A_{n, n}$, we see that (15) holds whenever $i$ or $j$ is equal to $n$.

It remains to check $f_{i j}$ for $1 \leq i, j \leq n-1$. From (11), we see that

$$
f_{i j}=(-1)^{i+j}\left|A_{j, i}\right||B|-(-1)^{i+j}\left|B_{j, i}\right||A|
$$

For these values of $i$ and $j$ the equation (15) follows immediately by applying Lemma 1 
below to (16). This completes the proof of Theorem 2.

Lemma 1. Let $1 \leq i, j \leq n-1$. Then

$$
|A|\left|B_{j, i}\right|=\left|A_{j, i}\right||B|-\left|A_{n, i}\right|\left|A_{j, n}\right|
$$

Proof. Lemma 1 is a classical formula of Jacobi (1833), sometimes called the Dodgson Condensation Formula.

To apply this general Theorem 2 in the proofs of Theorem 1 we set $S^{\prime}=S \cup\{j\}, A=\gamma_{S^{\prime}}$, $B=\gamma_{S}, \mathbf{x}^{\prime}=\mathbf{y}^{\prime}=d_{S^{\prime}}$ and $\mathbf{x}=\mathbf{y}=d_{S}$. Since $\gamma_{S^{\prime}}$ is symmetric, we have $|R|=|C|$ in this case. Furthermore, if we set $q_{S^{\prime}}=\left(\gamma_{S^{\prime}}\right)^{-1} d_{S^{\prime}}$, then by Cramer's Rule, we have $q_{S_{j}^{\prime}}=\frac{|R|}{\left|\gamma_{S^{\prime}}\right|}$. Therefore we obtain the following.

Corollary 1. With the notation above,

$$
\left|\gamma_{S}\right| d_{S^{\prime}}^{T} \operatorname{Adj}\left[\gamma_{S^{\prime}}\right] d_{S^{\prime}}-\left|\gamma_{S^{\prime}}\right| d_{S}^{T} \operatorname{Adj}\left[\gamma_{S}\right] d_{S}=\left(\left|\gamma_{S^{\prime}}\right| q_{S_{j}^{\prime}}\right)^{2}
$$

For the proof of Theorem 1 the corollary yields

$$
\Pi_{S^{\prime}}-\Pi_{S}=\frac{\left|\gamma_{S}\right| d_{S^{\prime}}^{T} \operatorname{Adj}\left[\gamma_{S^{\prime}}\right] d_{S^{\prime}}-\left|\gamma_{S^{\prime}}\right| d_{S}^{T} \operatorname{Adj}\left[\gamma_{S}\right] d_{S}}{\left|\gamma_{S}\right|\left|\gamma_{S^{\prime}}\right|}=\frac{\left|\gamma_{S^{\prime}}\right|}{\left|\gamma_{S}\right|}\left(q_{S_{j}^{\prime}}\right)^{2}
$$


Table 1. Variable Notation

SP Single-function product

FP Fusion product

$p_{k} \quad$ Price of product $k$

$q_{k} \quad$ Quantity of product $k$ offered by the firm (decision variable)

$a_{k} \quad$ Market potential (the maximum amount of willingness-to-pay) of product $k$

$c_{k} \quad$ Unit variable cost of product $k$

$d_{k} \quad$ Scaled profit margin of product $k, d_{k}=\frac{a_{k}-c_{k}}{2}$

$S \quad$ The optimal product portfolio with $s$ distinct products

$s \quad$ The cardinality of the optimal product portfolio $S$

$r_{k, j} \quad$ Substitution index representing one unit of product $j$ on the price of product $k$

$\gamma_{k, j} \quad$ Average substitution index between products $k$ and $j$

$\Pi_{S} \quad$ Profit function of portfolio $S$

$K \quad$ A subset of single-function products associate with product portfolio $S$

$\lambda_{K} \quad$ Linear combination of profit margins from all SPs in $K$

$\theta_{K} \quad$ Concavity index composed of the substitution indices of all SPs in $K$

NFPS No fusion product strategy

APS All product strategy

PFPS Partial fusion product strategy

SFPS Single fusion product strategy 
Table 2. Matrix A

\begin{tabular}{crrrrrrr}
\hline$\gamma_{i, j}^{A}$ & 1 & 2 & 3 & 4 & 5 & 6 & 7 \\
\hline 1 & 1 & 0 & 0 & 0.8 & 0.8 & 0 & 0.8 \\
2 & 0 & 1 & 0 & 0.6 & 0 & 0.6 & 0.6 \\
3 & 0 & 0 & 1 & 0 & 0.9 & 0.9 & 0.9 \\
4 & 0.8 & 0.6 & 0 & 1 & 0.85 & 0.85 & 0.8 \\
5 & 0.8 & 0 & 0.9 & 0.85 & 1 & 0.9 & 0.95 \\
6 & 0 & 0.6 & 0.9 & 0.85 & 0.9 & 1 & 0.95 \\
7 & 0.8 & 0.6 & 0.9 & 0.8 & 0.95 & 0.95 & 1 \\
\hline
\end{tabular}


Table 3. Matrix B

\begin{tabular}{crrrrrrr}
\multicolumn{2}{l}{$r$} \\
\hline$\gamma_{i, j}^{B}$ & 1 & 2 & 3 & 4 & 5 & 6 & 7 \\
\hline 1 & 1 & 0 & 0 & 0.5 & 0.5 & 0 & 0.5 \\
2 & 0 & 1 & 0 & 0.6 & 0 & 0.6 & 0.6 \\
3 & 0 & 0 & 1 & 0 & 0.9 & 0.9 & 0.9 \\
4 & 0.5 & 0.6 & 0 & 1 & 0.6 & 0.7 & 0.7 \\
5 & 0.5 & 0 & 0.9 & 0.6 & 1 & 0.9 & 0.95 \\
6 & 0 & 0.6 & 0.9 & 0.7 & 0.9 & 1 & 0.95 \\
7 & 0.5 & 0.6 & 0.9 & 0.7 & 0.95 & 0.95 & 1 \\
\hline
\end{tabular}


Table 4 . Changing the profit margins $\left(d_{1}\right.$ and $\left.d_{2}\right)$ and the results.

\begin{tabular}{cccccccccccc}
\hline Case & $\gamma$ & $d_{1}$ & $d_{2}$ & $d_{3}$ & $d_{4}$ & $d_{5}$ & $d_{6}$ & $d_{7}$ & Opt. Portfolio & $q^{*}$ & Profit \\
\hline 1 & $\gamma_{i, j}^{A}$ & 24 & 38 & 38 & 50 & 53 & 58 & 67 & $\{7\}$ & $\{67\}$ & $\$ 4489$ \\
2 & $\gamma_{i, j}^{A}$ & 30 & 38 & 38 & 50 & 53 & 58 & 67 & $\{7\}$ & $\{67\}$ & $\$ 4489$ \\
3 & $\gamma_{i, j}^{A}$ & 24 & 38 & 44 & 50 & 53 & 58 & 67 & $\{2,3,4\}$ & $\{12.5,44,42.5\}$ & $\$ 4536$ \\
4 & $\gamma_{i, j}^{B}$ & 24 & 38 & 38 & 50 & 53 & 58 & 67 & $\{4,7\}$ & $\{6,62.7\}$ & $\$ 4507$ \\
5 & $\gamma_{i, j}^{B}$ & 24 & 44 & 38 & 50 & 53 & 58 & 67 & $\{2,5\}$ & $\{44,53\}$ & $\$ 4745$ \\
6 & $\gamma_{i, j}^{B}$ & 24 & 38 & 44 & 50 & 53 & 58 & 67 & $\{1,2,3,4\}$ & $\{4.5,14.6,44,39\}$ & $\$ 4548$ \\
\hline
\end{tabular}

